\title{
LATTICES WHOSE IDEAL LATTICE IS STONE
}

\author{
by R. BEAZER
}

(Received 1st December 1981)

\section{Introduction}

An elementary fact about ideal lattices of bounded distributive lattices is that they belong to the equational class $\mathscr{B}_{\omega}$ of all distributive $p$-algebras (distributive lattices with pseudocomplementation). The lattice of equational subclasses of $\mathscr{B}_{\omega}$ is known to be a chain

$$
\mathscr{B}_{0} \subset \mathscr{B}_{1} \subset \cdots \subset \mathscr{B}_{n} \subset \cdots \subset \mathscr{B}_{\omega}
$$

of type $\omega+1$, where $\mathscr{B}_{0}$ is the class of Boolean algebras and $\mathscr{B}_{1}$ is the class of Stone algebras. G. Grätzer in his book [7] asks after a characterisation of those bounded distributive lattices whose ideal lattice belongs to $\mathscr{B}(n \geqq 1)$. The answer to the problem for the case $n=0$ is well known: the ideal lattice of a bounded lattice $L$ is Boolean if and only if $L$ is a finite Boolean algebra. D. Thomas [10] recently solved the problem for the case $n=1$ utilising the order-topological duality theory for bounded distributive lattices and in [5] W. Bowen obtained another proof of Thomas's result via a construction of the dual space of the ideal lattice of a bounded distributive lattice from its dual space. In this paper we give a short, purely algebraic proof of Thomas's result and deduce from it necessary and sufficient conditions for the ideal lattice of a bounded distributive lattice to be a relative Stone algebra. Grätzer's problem for the case $n=1$ can be paraphrased as: Characterise those bounded distributive lattices whose congruence kernels form a Stone algebra. We ask and answer the same question for distributive $p$ algebras and distributive double $p$-algebras drawing from the main result a characterisation of those double Heyting algebras whose congruence lattice is Stone.

\section{Preliminaries}

Let $\langle L, \vee, \wedge, 0,1\rangle$, henceforth simply $L$, be a bounded distributive lattice. Throughout, we shall write $\operatorname{Cen}(L)$ for the centre of $L, \mathscr{I}(L)$ for its ideal lattice and $L / I$ for $L / \theta(I)$, where $\theta(I)$ is the principal congruence of $L$ generated by $I \in \mathscr{I}(L)$. If $L$ is equipped with a unary operation * characterised by the property:

$$
a \wedge x=0 \text { if and only if } x \leqq a^{*}
$$

then $L$ is called a distributive p-algebra or distributive lattice with pseudocomplementation. 
If, in any such algebra, we write $B(L)=\left\{x \in L ; x=x^{* *}\right\}$ then $\left\langle B(L), \cup, \wedge,{ }^{*}, 0,1\right\rangle$ is a Boolean algebra, called the skeleton of $L$, when the join operation $\cup$ is defined on $B(L)$ by $a \cup b=(a \vee b)^{* *}$ and $D(L)=\left\{x \in L ; x^{*}=0\right\}$ is a filter in $L$, called the dense filter. A Stone algebra is a distributive $p$-algebra satisfying the identity $x^{*} \vee x^{* *}=1$ and a relative Stone algebra is a bounded lattice in which every interval is a Stone algebra. Relative Stone algebras are intimately related to Heyting algebras; this is, bounded (distributive) lattices equipped with a binary operation $*$ characterised by the property:

$$
a \wedge x \leqq b \text { if and only if } x \leqq a * b
$$

Indeed, the classes of relative Stone algebras and Heyting algebras satisfying the identity $(x * y) \vee(y * x)=1$ are coincident. A distributive $p$-algebra endowed with a unary operation ${ }^{+}$characterised by the property dual to that for $*$ is called a distributive double p-algebra and a Heyting algebra endowed with a binary operation + characterised by the property dual to that for $*$ is called a double Heyting algebra. If $L$ is a distributive double $p$-algebra and $a \in L$ then elements $a^{n(*+)}(n<\omega)$ can be defined recursively by

$$
a^{0(*+)}=a, a^{(k+1)(*+)}=a^{k(*+) *+}
$$

Elements $a^{n(+*)}(n<\omega)$ can also be defined in a similar fashion and the following are known to hold (see [2]):

$$
x \leqq x^{*+},(x \vee y)^{*+}=x^{*+} \vee y^{*+}, \operatorname{Cen}(L)=\left\{x \in L ; x=x^{*+}\right\}
$$

By a congruence relation on a distributive $p$-algebra, distributive double $p$-algebra, double Heyting algebra we mean a lattice congruence preserving ${ }^{*},{ }^{*}$ and ${ }^{+}, *$ and + , respectively, and by a congruence kernel we mean any congruence class containing 0 .

All undefined terms as well as general lattice theoretic results and facts about distributive $p$-algebras may be found in [1] or [7].

\section{Grätzer's problem for $n=1$}

The key to the solution of the problem is the following simple observation:

Lemma 1. An ideal $I$ in a bounded distributive lattice $L$ is complemented if and only if it is of the form $(z]$, for some $z \in \operatorname{Cen}(L)$.

Proof. Clearly, if $z \in \operatorname{Cen}(L)$ then $(z]$ has complement $\left(z^{\prime}\right]$ in $\mathscr{I}(L)$. Conversely, if $I \in \operatorname{Cen}(\mathscr{I}(L))$ then $I \vee I^{*}=L$ so that $1=z \vee w$, for some $z \in I, w \in I^{*}$ which since $I^{*}=\{x \in L ; x \wedge i=0$ for all $i \in I\}$, shows that $z \in \operatorname{Cen}(L)$ and $z^{\prime}=w$. For any $x \in I^{*}$, we have $x \wedge z=0$ so that $x \leqq z^{*}=z^{\prime}=w$ and, therefore, $I^{*} \subseteq(w]$. Thus, $I^{*}=(w]$, since $w \in I^{*}$, and it follows that $I=(z]$.

Theorem 2. The ideal lattice of a bounded distributive lattice $L$ is a Stone algebra if and only if $L$ is a Stone algebra whose centre is complete. 
Proof. If $\mathscr{I}(L)$ is Stone than $(a]^{*} \in \operatorname{Cen}(\mathscr{I}(L))$, for any $a \in L$, and so $(a]^{*}=(z]$, for some $z \in \operatorname{Cen}(L)$, by Lemma 1. However, $(a]^{*}=\{x \in L ; x \wedge a=0\}$ and so $a^{*}$ exists and belongs to $\operatorname{Cen}(L)$, for any $a \in L$. In other words, $L$ is a Stone algebra. In order to show that $\operatorname{Cen}(L)$ is a complete lattice, it is enough by Lemma 1 to show that if $Z \subseteq \operatorname{Cen}(L)$ then $\bigcap\{(z] ; z \in Z\}$ is of the form $I^{*}$, for some $I \in \mathscr{I}(L)$. We claim that $I=\bigvee\left\{\left(z^{\prime}\right] ; z \in Z\right\}$ is an ideal satisfying our needs. Indeed, $x \in I^{*}$ if and only if $x \wedge a=0$, for all $a \in \bigvee\left\{\left(z^{\prime}\right]\right.$; $z \in Z\}$, and by distributivity this is equivalent to $x \wedge z^{\prime}=0$, for all $z \in Z$, since $\bigvee\left\{\left(z^{\prime}\right]\right.$; $z \in Z\}$ consists of all finite joins of elements in the set union $\bigcup\left\{\left(z^{\prime}\right] ; z \in Z\right\}$. This, in turn, holds if and only if $x \in \bigcap\{(z] ; z \in Z\}$. Thus, $I^{*}=\bigcap\{(z] ; z \in Z\}$.

Conversely, suppose that $L$ is a Stone algebra and that $\operatorname{Cen}(L)$ is complete. In order to show that $\mathscr{I}(L)$ is Stone it is enough to show that $I^{*} \in \operatorname{Cen} \mathscr{I}(L)$, for any $I \in \mathscr{I}(L)$. First, observe that $i^{*} \in \operatorname{Cen}(L)$ for any $i \in I$, since $L$ is Stone, and so the existence of $z$ $=\bigwedge\left\{i^{*} ; i \in I\right\}$, taken in $\operatorname{Cen}(L)$, is guaranteed. We claim that $I^{*}=(z]$. Indeed, $x \in I^{*}$ if and only if $x$ is a lower bound in $L$ of $\left\{i^{*} ; i \in I\right\}$ or, equivalently, $x^{* *}$ is a lower bound in $L$ of $\left\{i^{*} ; i \in I\right\}$. This, in turn, is equivalent to $x \leqq z$, since $x \leqq x^{* *} \in \operatorname{Cen}(L)$ and $z^{* *}$ $=z \in \operatorname{Cen}(L)$. Thus, $I^{*}=(z] \in \operatorname{Cen}(\mathscr{I}(L))$.

Corollary 3. For a bounded distributive lattice $L$, the following are equivalent:

(i) $\mathscr{I}(L)$ is a relative Stone algebra,

(ii) For any $I \in \mathscr{I}(L), L / I$ is a Stone algebra whose centre is complete.

(iii) $L$ is a relative Stone algebra whose centre is complete and, for any $I \in D(\mathscr{I}(L)), L / I$ is a Stone algebra whose centre is complete.

Proof. It is well known (see [1]) that for a bounded distributive lattice $L$ to be relative Stone it is necessary and sufficient that every principal filter of $L$ be a Stone algebra. This fact, applied to $\mathscr{I}(L)$ in conjunction with Theorem 2 and the equally well known fact that $\mathscr{I}(L / I) \cong[I)$, for any $I \in \mathscr{I}(L)$, establishes the equivalence of (i) and (ii). Now, if $\mathscr{I}(L)$ is relative Stone and $b \in L$ is arbitrary then $L /(b]$ is a Stone algebra. In particular, it follows that, given any $a \in L$, there exists $\bar{a} \in L$ such that

$$
[a]_{b} \wedge[x]_{b}=[0]_{b} \text { if and only if }[x]_{b} \leqq[\bar{a}]_{b}
$$

where $[x]_{b}$ denotes the congruence class of $\theta((b])$ containing $x$. As a consequence of this and the well known description of principal congruences on distributive lattices we conclude that

$$
a \wedge x \leqq b \quad \text { if and only if } \quad x \leqq \bar{a} \vee b
$$

and so $L$ is a Heyting algebra in which $a * b=\bar{a} \vee b$, for any $a, b \in L$. Furthermore, the identity $(a * b) \vee(b * a)=1$ holds in $L$ by virtue of the fact that it holds in $\mathscr{I}(L)$. Indeed, for any $I, J \in \mathscr{I}(L)$, we have $I * J=\{x \in L ; x \wedge i \in J$, for all $i \in I\}$ and so, in particular, $(a] *(b]=(a * b]$, for any $a, b \in L$. Therefore, $(a * b] \vee(b * a]=L$ and so $(a * b) \vee(b * a)=1$. Thus, $L$ is relative Stone and the proof that (ii) implies (iii) is complete. Moreover, condition (iii) in conjunction with Theorem 2 shows that $\mathscr{I}(L / I)$ and therefore $[I)$ is a Stone algebra, for any $I \in D(\mathscr{I}(L))$. Thus, (iii) implies (i), since it is well known that for a 
Stone algebra to be relative Stone it is necessary and sufficient that each of its principal filters generated by a dense element be a Stone algebra (see [1]).

Corollary 4. The congruence lattice of a Boolean lattice $L$ is relative Stone if and only if every homomorphic image of $L$ is complete.

In connection with Corollaries 3 and 4 we point out that if $L$ is a Stone algebra whose centre is complete and $I \in \mathscr{I}(L)$ the $L / I$ is not necessarily Stone nor is its centre necessarily complete. Indeed, if $L$ is the Stone algebra obtained by adjoining a new zero and unit to the four-element Boolean algebra and $I$ is the principal ideal of $L$ generated by its only atom then $L / I$ is isomorphic to the four-element Boolean algebra with a new unit adjoined and so is not Stone. Furthermore, while the field of all subsets of an uncountable set $X$ is complete, its quotient modulo the ideal of all countable subsets of $X$ is not.

\section{The problem for distributive $p$-algebras and double $p$-algebras}

Earlier we pointed out that a subset of a bounded distributive lattice is an ideal if and only if it is a congruence kernel. W. Cornish [6] showed that an ideal $I$ in a distributive $p$-algebra is a congruence kernel if and only if $i^{* *} \in I$ whenever $i \in I$. In addition, Cornish showed that the lattice of congruence kernels of a distributive $p$-algebra $L$ is isomorphic to the ideal lattice of the skeleton $B(L)$ of $L$. T. S. Blyth [4] showed that exactly the same is true for pseudo-complemented semilattices with, of course, the appropriate definition of congruence kernel in this context. Thus, we have

Theorem 5. The lattice of congruence kernels of a pseudo-complemented semilattice or of a distributive p-algebra $L$ is a Stone algebra if and only if the skeleton of $L$ is complete.

The situation for distributive double $p$-algebras is not so simple but nevertheless tractible. It follows on dualising results in [2] that a subset $I$ of a distributive double $p$ algebra is a congruence kernel if and only if $i^{*+} \in I$ whenever $i \in I$. Moreover, it is easy to show, utilising the well known description of infinite joins in ideal lattices of distributive lattices and the identity $(x \vee y)^{*+}=x^{*+} \vee y^{*+}$ which holds in any distributive double p-algebra, that the lattice $K(L)$ of congruence kernels of a distributive double $p$-algebra $L$ is a complete sublattice of the ideal lattice $\mathscr{I}(L)$ of $L$. Consequently, $K(L) \in \mathscr{B}_{\omega}$ and, using Lemma 1 , it is easy to see that $\operatorname{Cen}(K(L))=\{(z]$; $z \in \operatorname{Cen}(L)\}$. When, then, is $K(L)$ Stone?

Theorem 6. The lattice $K(L)$ of congruence kernels of a distributive double p-algebra $L$ is a Stone algebra if and only if $\bigwedge_{n<\omega} a^{n(+*)}$ and $\bigwedge S$ exist in $L$, for any $a \in B(L)$ and $S \subseteq \operatorname{Cen}(L)$.

Proof. We start with the observation that if $a \in L$ and $I(a)=\left\{x \in L ; x \leqq a^{n(*+)}\right.$, for some $n<\omega\}$ then $I(a) \in K(L)$, since $a^{n(*+)} \leqq a^{m(*+)}$ whenever $n \leqq m$, and claim that $I(a)^{*}$ $=\bigcap_{n<\omega}\left(a^{* n(+*)}\right]$. With the aim of showing that the ideal $\bigcap_{n<\omega}\left(a^{* n(+*)}\right] \in K(L)$, let 
$x \in \bigcap_{n<\omega}\left(a^{* n(+*)}\right]$ and let $k<\omega$. Then $x \leqq a^{*(k+1)(+*)}$ so that $x^{*} \geqq a^{(k+1)(*+) * *} \geqq a^{(k+1)(*+)}$ and, therefore, $x^{*+} \leqq a^{(k+1)(*+)+}=a^{* k(+*)++} \leqq a^{* k(+*)}$. Thus, $x^{*+} \in \bigcap_{n<\omega}\left(a^{* n(+*)}\right]$. Moreover, if $x \in I(a) \cap \bigcap_{n<\omega}\left(a^{* n(+*)}\right]$ then $x \leqq a^{n(*+)}$, for some $n<\omega$, and $x \leqq a^{* n(+*)}=a^{n(*+) *}$ so that $x \leqq a^{n(*+)} \wedge a^{n(*+) *}=0$. Therefore, $I(a) \cap \bigcap_{n<\omega}\left(a^{* n(+*)}\right]=\{0\}$. In addition, if $K \in K(L)$ satisfies $I(a) \cap K=\{0\}$ and $k \in K$ then $k \wedge a^{n(*+)}=0$ for all $n<\omega$; that is, $k \leqq a^{n(*+) *}=a^{* n(+*)}$ for all $n<\omega$. Thus, $K \subseteq \bigcap_{n<\omega}\left(a^{* n(+*)}\right]$ and we conclude that $I^{*}=\bigcap_{n<\omega}\left(a^{* n(+*)}\right]$. It follows, now, that if $K(L)$ is a Stone algebra and $a \in L$ then $I(a)^{*} \in \operatorname{Cen}(K(L))$ and so $I(a)^{*}=(z]$, for some $z \in \operatorname{Cen}(L)$. Thus, $\bigwedge_{n<\omega} a^{* n(+*)}$ exists, for any $a \in L$. Equivalently, $\bigwedge_{n<\omega} a^{n(+*)}$ exists, for any $a \in B(L)$. Next, it is straightforward to show that if $S \subseteq \operatorname{Cen}(L)$ and $I\left(S^{\prime}\right)=\left\{x \in L ; x \leqq s_{1}^{\prime} \vee \cdots \vee s_{n}^{\prime}\right.$, for some $\left.s_{i} \in S, 1 \leqq i \leqq n\right\}$ then $I\left(S^{\prime}\right) \in K(L)$. Moreover, by distributivity, $I \in K(L)$ satisfies $I \cap I\left(S^{\prime}\right)$ $=\{0\}$ if and only if $i \wedge s^{\prime}=0$, for all $i \in I$ and $s \in S$ : equivalently, if and only if $I \subseteq \bigcap\{(s] ; s \in S\}$. Consequently, $I\left(S^{\prime}\right)^{*}=\bigcap\{(s] ; s \in S\}$ which, since $I\left(S^{\prime}\right)^{*}=(z]$ for some $z \in \operatorname{Cen}(L)$, shows that $\bigwedge S$ exists, for any $S \subseteq \operatorname{Cen}(L)$.

Conversely, suppose that, for any $a \in B(L)$ and $S \subseteq \operatorname{Cen}(L), \bigwedge_{n<\omega} a^{n+*)}$ and $\bigwedge S$ exist in $L$. We start by showing that all such meets necessarily belong to Cen $(L)$. Indeed, if $a \in B(L), k<\omega$ and $m(a)=\bigwedge_{n<\omega} a^{n(+*)}$ then $m(a) \leqq a^{(k+1)(+*)}$ so that $m(a)^{*} \geqq a^{(k+1)(+*) *}$ $=a^{k(+*)+* *} \geqq a^{k(+*)+}$ and, therefore, $m(a)^{*+} \leqq a^{k(+*)++} \leqq a^{k(+*)}$. It follows that $m(a)^{*+} \leqq m(a)$ and so $m(a) \in \operatorname{Cen}(L)$, since $x \leqq x^{*+}$ holds for any $x \in L$. Moreover, if $S \subseteq \operatorname{Cen}(L)$ and $m(S)=\bigwedge S$ then, since $m(S) \leqq s$ implies $m^{*+}(S) \leqq s$, for any $s \in S$, we have $m^{*+}(S) \leqq m(S)$ and so $m(S) \in \operatorname{Cen}(L)$. It follows, now, that if $i \in I \in K(L)$ then $m\left(i^{*}\right)$ and, therefore, $z=\bigwedge\left\{m\left(i^{*}\right) ; i \in I\right\}$ exists and is central. We claim that $I^{*}=(z]$. Indeed, if $x \in I^{*}$, $i \in I$ and $n<\omega$ then $x \wedge i^{n(*+)}=0$ so that $x \leqq i^{n(*+) *}=i^{* n(+*)}$. Therefore, $x \leqq m\left(i^{*}\right)$, for all $i \in I$, and so $x \leqq z$. Hence, $I^{*} \subseteq(z]$. For the reverse inclusion, first observe that if $x \leqq z$, $i \in I$ and $m<\omega$ then $x^{m(*+)} \leqq z^{m(*+)}=z \leqq i^{*}$, since $z \in \operatorname{Cen}(L)$, and so $i \leqq i^{* *} \leqq x^{m(*+) *}$. It follows, now, that $I(x) \cap I=\{0\}$; because if $i \in I(x) \cap I$ then $i \leqq x^{n(*+)}$, for some $n<\omega$, and so $i \leqq x^{n(*+)} \wedge x^{n(*+) *}=0$. Therefore, $x \in I(x) \subseteq I^{*}$. Thus, we have shown that $I^{*}$ is complemented in $K(L)$, for any $I \in K(L)$. Equivalently, $K(L)$ is a Stone algebra.

In [9] P. Köhler call $s$ an ideal $I$ in a double Heyting algebra $L$ normal if $a \in I$ implies $a^{*+} \in I$ and proves that the congruence lattice of $L$ is isomorphic to the lattice of normal ideals of $L$. Thus, we have

Corollary 7. The congruence lattice of a double Heyting algebra $L$ is a Stone algebra if and only if $\bigwedge_{n<\omega} a^{n(+*)}$ and $\bigwedge S$ exist, for any $a \in B(L)$ and $S \subseteq \operatorname{Cen}(L)$.

A double $p$-algebra is called regular if it satisfies the identity $\left(x \wedge x^{+}\right) \vee\left(y \vee y^{*}\right)$ $=y \vee y^{*}$. In [8], T. Katriňák shows that a regular double $p$-algebra is, in fact, a double Heyting algebra in which $x * y$ and $x+y$ are double $p$-algebra polynomials. As a consequence, double Heyting algebra congruences and double $p$-algebra congruences coincide for regular double $p$-algebras. Thus, Corollary 7 is a generalisation and, simultaneously, an improvement of the main result of [3].

Added in proof. Recently, I learnt that Theorem 2 was proved by T. Katrinák in his paper "Notes on Stone lattices I", Mat. Casopsis Sloven. Akad. Vied. 16 (1966), 128142 (in Russian). A version of Theorem 2 for join similattices with 1 appears in his paper "Pseudocomplementäre Halbverbände", Mat. Casopsis Sloven. Akad. Vied. 18 (1968), 121-143. 


\section{REFERENCES}

1. R. Balbes and Ph. Dwinger, Distributive Lattices (Univ. Missouri Press, Columbia, Missouri, 1974).

2. R. BEAzER, The determination congruence on double p-algebras, Algebra Universalis 6 (1976), 121-129.

3. R. Beazer, Regular double p-algebras with Stone congruence lattices, Algebra Universalis 9 (1979), 238-243.

4. T. S. BLyth, Ideals and filters of pseudo-complemented semilattices, Proc. Edinburgh Math. Soc. 23 (1980), 301-316.

5. W. Bowen, Lattice Theory and Topology (Ph.D. thesis, Oxford, 1981).

6. W. Cornish, Congruences on distributive pseudocomplemented lattices, Bull. Austral. Math. Soc. 8 (1973), 161-179.

7. G. Grätzer, General Lattice Theory (Birkhäuser Verlag, Basel, 1978).

8. T. Katriñá, The structure of distributive double $p$-algebras. Regularity and congruences, Algebra Universalis 3 (1973), 238-246.

9. P. KÖhleR, A subdirectly irreducible double Heyting algebra which is not simple, Algebra Universalis 10 (1980), 189-194.

10. D. Thомаs, Problems in Functional Analysis (Ph.D. thesis, Oxford, 1976).

Department of Mathematics

UNIVERSITY OF GLASGOW

GLASGOW G12 8QW 